\title{
Prognostic roles of the expression of sphingosine-1-phosphate metabolism enzymes in non-small cell lung cancer
}

\author{
Yingqin Wang ${ }^{1 \#}$, Yaxing Shen ${ }^{2 \#}$, Xia Sun ${ }^{3,4}$, Tinah L. Hong ${ }^{5}$, Long Shuang Huang ${ }^{6}$, Ming Zhong ${ }^{1}$ \\ ${ }^{1}$ Department of Critical Care Medicine, ${ }^{2}$ Department of Thoracic Surgery, Zhongshan Hospital, Fudan University, Shanghai 200032, China; \\ ${ }^{3}$ Department of Nephrology, Xuzhou Municipal Hospital Affiliated to Xuzhou Medical University, Xuzhou 221000, China; ${ }^{4}$ Cancer Institute, \\ Xuzhou Medical University, Xuzhou 221002, China; ${ }^{5}$ New Trier High School, Winnetka, IL, USA; ${ }^{6}$ Department of Pharmacology, Shanghai \\ Hospital of Traditional Chinese Medicine, Shanghai 200032, China \\ Contributions: (I) Conception and design: M Zhong, LS Huang; (II) Administrative support: M Zhong; (III) Provision of study materials or patients: \\ LS Huang, Y Wang; (IV) Collection and assembly of data: Y Wang, Y Shen, X Sun; (V) Data analysis and interpretation: Y Wang, Y Shen, TL Hong, \\ X Sun; (VI) Manuscript writing: All authors; (VII) Final approval of manuscript: All authors. \\ "These authors contributed equally to this work. \\ Correspondence to: Ming Zhong, MD, PhD. Department of Critical Care Medicine, Zhongshan Hospital, Fudan University, 180 Fenglin Road, \\ Shanghai 200032, China. Email: zhong_ming@fudan.edu.cn; Long Shuang Huang, PhD. Department of Pharmacology, Shanghai Hospital of \\ Traditional Chinese Medicine, 274 Zhijiang Middle Rd, Zhabei Qu, Shanghai 200072, China. Email: lhuang82@uic.edu.
}

Background: Sphingosine-1-phosphate (S1P), a bioactive lipid, is generally increased in human non-
small cell lung cancer (NSCLC). Evidence has shown that the levels of enzymes in S1P metabolism were
associated with clinical outcomes in patients with NSCLC. Nevertheless, the roles of mRNA expression
of major enzymes (SPHK1, SPHK2 and SGPL1) in S1P metabolism for predicting outcomes in NSCLC
patients have not been determined.
Methods: "The Kaplan-Meier plotter" (the KM plotter) is an online database which contains gene
expression and clinical data of 1,928 NSCLC patients. In this study, we analyzed the relationship between
mRNA expression of major enzymes in S1P metabolism and overall survival (OS) in 1,926 NSCLC patients
with the KM plotter. Further analyses stratified by smoking history, non-metastasis patents, clinical stages,
negative surgical margin, chemotherapy and radiotherapy were also performed.
Results: High SPHK1 mRNA expression [hazard ratio (HR) 1.47, 95\% confident interval (CI): 1.28-1.68,
P=2.6e-08] was significantly correlated to worse OS, but high SPHK2 (0.66, 95\% CI: 0.59-0.75, P=1.9e-10)
or SGPL1 (HR 0.64, 95\% CI: $0.55-0.75$, P=8.7e-09) mRNA expression was in favor of better OS in NSCLC
patients.

Conclusions: The mRNA expression of SPHK1, SPHK2, and SGPL1 is potential predictor of outcomes in NSCLC patients.

Keywords: Sphingosine-1-phosphate (S1P); RNA, messenger; carcinoma; non-small cell lung cancer (NSCLC)

Submitted Feb 03, 2019. Accepted for publication Sep 23, 2019.

doi: 10.21037/tlcr.2019.10.04

View this article at: http://dx.doi.org/10.21037/tlcr.2019.10.04

\section{Introduction}

Lung cancer is one of the most common and deadly tumors, contributing to 1.8 million new cancer cases and 1.6 million cancer-related deaths worldwide every year (1). Non-small cell lung cancer (NSCLC) is the most common type of lung cancer, and accounts for $85 \%$ of all lung cancer (2). Lung adenocarcinoma (Ade) and lung squamous cell carcinoma (SCC) are major types of NSCLC, representing almost 50\% and $35 \%$ of NSCLC cases, respectively. Despite progress in treatments for NSCLC, most patients present with advanced stage and a poor five-year survival rate (3). Thus, further exploration of the potential prognostic biomarkers 
and therapeutic targets in NSCLC is still a priority.

Sphingosine-1-phosphate ( $\mathrm{S} 1 \mathrm{P})$ is a bioactive lipid generated from sphingosine by phosphorylation catalyzed by two distinct sphingosine kinase isoforms, SPHK1, and SPHK2 (4). S1P can be reversibly dephosphorylated by $\mathrm{S} 1 \mathrm{P}$ phosphatases and lipid phosphate phosphatases or irreversibly degraded by S1P lyase (SGPL1) $(4,5)$. S1P exerts most of its effects by autocrine or paracrine. Intracellular S1P is transported out by ATP-binding cassette transporters (ABC transporters) and spinster homolog 2 (Spns2) and binds to five membrane G-protein-coupled receptors, S1PR1-5 (4). The intracellular effects of S1P are independent of S1PRs (6). S1P plays pivotal roles in many physiological processes, including cell growth, migration, autophagy, angiogenesis, and survival (6). Previous studies have linked aberrant S1P signaling to tumor progression, invasion, metastasis, chemo- and radio-resistance in NSCLC $(4,7,8)$. It was reported that the levels of sphingosine kinases were correlated with clinical outcomes of NSCLC patients $(9,10)$. However, the prognostic roles of mRNA expression of SPHK1, SPHK2 and SGPL1 in NSCLC patients have not been determined.

The "Kaplan-Meier plotter" (KM plotter) is a widely used online database, which contains data from Cancer Biomedical Informatics Grid (caBIG), the Gene Expression Omnibus (GEO), and The Cancer Genome Atlas (TCGA) lung cancer datasets. Gene expression and clinical data are integrated simultaneously by a PostgreSQL server (11). The $\mathrm{KM}$ plotter has been used for identifying genes as potential prognostic markers or drug targets in various cancers, including gastric cancer $(12,13)$, liver cancer (13), breast cancer (14-18), ovarian cancer (19-21), and NSCLC (2224). In this study, we employed the KM plotter to analyze the prognostic roles of SPHK1, SPHK2, and SGPL1 mRNA expression in NSCLC patients.

\section{Methods}

The online public database, KM plotter (11), was used to analyze the association between specific gene mRNA expression and overall survival (OS). The KM plotter is an integrated platform identifying NSCLC patients with simultaneously available microarray gene expression data and published clinical characteristics, including survival, from TCGA lung cancer datasets, GEO, and caBIG (25). Currently, 1,928 NSCLC patients' gene expression profiles and survival data are available for analysis in the $\mathrm{KM}$ plotter. The follow-up times span of were 20 years. SPHK1,
SPHK2, and SGPL1 were entered into the database to obtain KM survival plots (http://kmplot.com/analysis/ index.php? $\mathrm{p}=$ service\& cancer=lung). The number-at-risk was indicated below the main plot. Affymetrix IDs were 219257_s_at (SPHK1), 40273_at (SPHK2), and 212322_ at (SGPL1). Auto- selected best cutoff value checkbox was selected for gene mRNA expression to divide patients into high expression and low expression groups. All possible cutoff values between the lower and upper quartiles were computed, and the best performing threshold was used as a cut-off. If the gene had multiple chipsets, the userselected probe set was selected for analysis. Histology, grade, stage, gender, and smoking history were selected for the Cox multivariate analysis. Hazard ratio (HR) with 95\% confidence intervals (CI) and log rank P were automatically calculated by the KM plotter.

\section{Results}

The mRNA expression of three major S1P metabolism enzymes (SPHK1, SPHK2 and SGPL1) in NSCLC patients can be found in the KM plotter database (www.kmplotter. com). The KM survival curves were drafted for all NSCLC patients $(\mathrm{n}=1926)$, Ade patients $(\mathrm{n}=720)$, and SCC patients $(\mathrm{n}=524)$, who were followed up for 20 years.

For SPHK1, the Affymetrix ID is 219257_s_at. High SPHK1 mRNA expression was significantly associated with worse OS in all NSCLC patients (HR 1.47, 95\% CI: 1.281.68, $\mathrm{P}=2.6 \mathrm{e}-08$ ) (Figure $1 A$ ) and Ade patients (HR 0.73, 95\% CI: 0.56-0.94, $\mathrm{P}=0.014$ ) (Figure $1 B$ ), but not in SCC patients (HR 1.32, 95\% CI: 0.95-1.84, $\mathrm{P}=0.094$ ) (Figure 1C).

For SPHK2, the Affymetrix ID is 40273_at. High SPHK2 mRNA expression was significantly linked to better OS in all NSCLC patients (HR 0.66, 95\% CI: 0.59-0.75, $\mathrm{P}=1.9 \mathrm{e}-10$ ) (Figure $2 A$ ) and Ade patients (HR 0.53, 95\% CI: 0.42-0.67, $\mathrm{P}=6.5 \mathrm{e}-08$ ) (Figure $2 B$ ), but not in SCC patients (HR 1.18, 95\% CI: 0.91-1.53, $\mathrm{P}=0.21$ ) (Figure 2C).

For SGPL1, the Affymetrix ID is 212322_at. High SGPL1 mRNA expression was significantly associated with better OS in all NSCLC patients (HR 0.64, 95\% CI: $0.55-$ 0.75, $\mathrm{P}=8.7 \mathrm{e}-09$ ) (Figure 3A), Ade patients (HR 0.45, 95\% CI: 0.34-0.6, $\mathrm{P}=2.3 \mathrm{e}-08$ ) (Figure $3 B$ ), and SCC patients (HR 0.76, 95\% CI: 0.6-0.97, $\mathrm{P}=0.024$ ) (Figure 3C).

We further assessed the prognostic value of these enzymes in S1P metabolism with other pathological features and clinical treatments: smoking history, metastasis patients, clinical stages, negative surgical margin, chemotherapy, and radiotherapy. Table 1 shows that high SPHK1, and SGPL1 

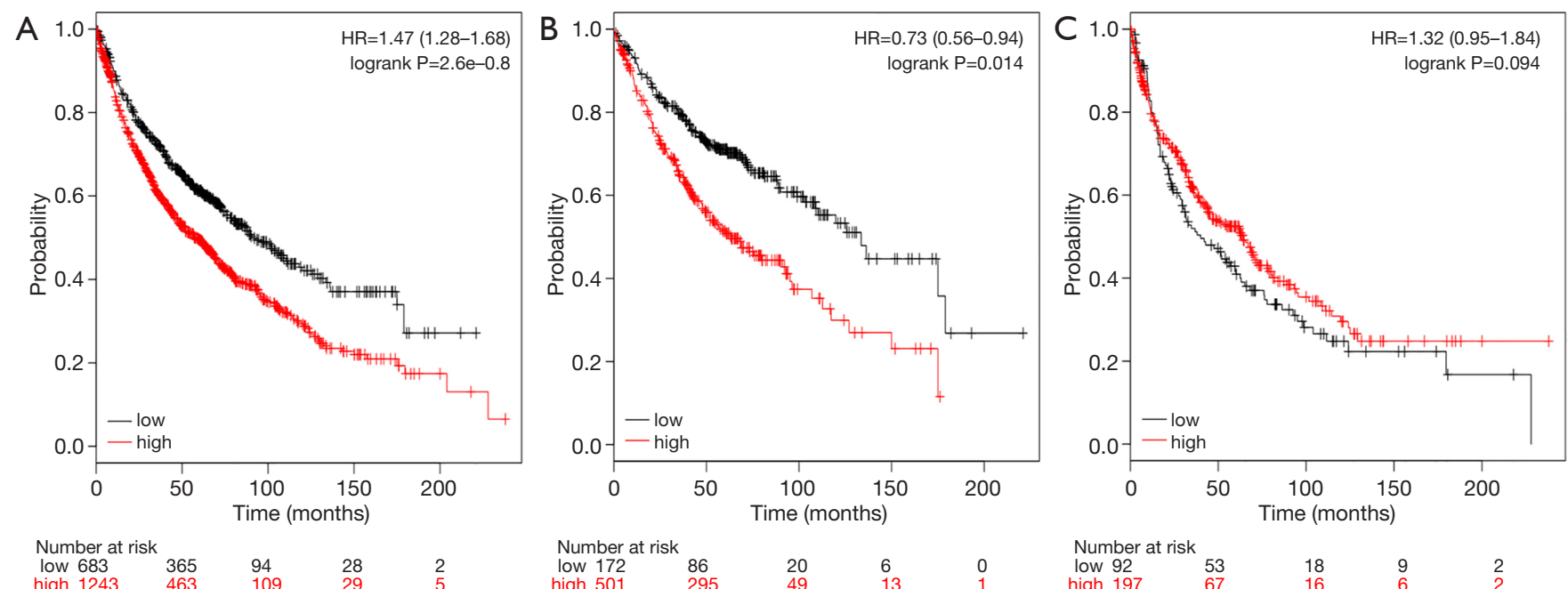

Figure 1 The prognostic role of SPHK1 mRNA level in NSCLC patients. The Affymetrix ID is 219257_s_at. The Kaplan-Meier survival curves are drafted for (A) all NSCLC patients $(n=1,926)$, (B) Ade patients $(n=720)$, and (C) SCC patients (n=524). NSCLC, non-small cell lung cancer; Ade, adenocarcinoma; SCC, squamous cell carcinoma.
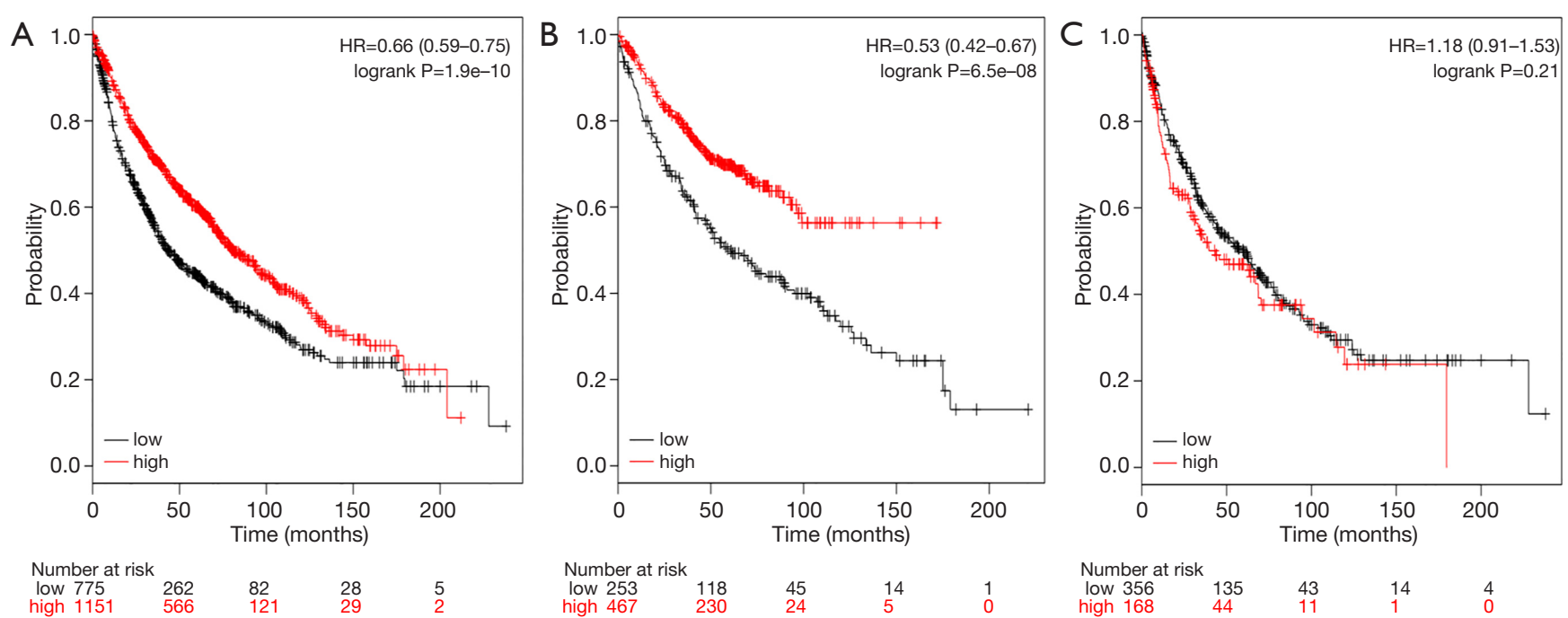

Figure 2 The prognostic role of SHPK2 mRNA level in NSCLC patients. The Affymetrix ID is 40273_at. The Kaplan-Meier survival curves are drafted for (A) all NSCLC patients $(n=1,926)$, (B) Ade patients $(n=720)$, and (C) SCC patients ( $n=524)$. NSCLC, non-small cell lung cancer; Ade, adenocarcinoma; SCC, squamous cell carcinoma.

mRNA expression was correlated with clinical outcomes in NSCLC patients, despite smoking history. High SPHK2 mRNA expression was only associated with better OS in patients who used to smoke. From Table 2 and Table 3, high SPHK1, SPHK2 and SGPL1 mRNA expression were associated with OS in patients without metastasis and with negative surgical margins. Table 4 shows that high SPHK1 mRNA expression was associated with clinical stage I NSCLC patients. High SPHK2 and SGPL1 mRNA expression were correlated with OS in clinical stage I and II NSCLC patients. Table 5 shows that high SPHK1 and SPHK2 mRNA expression was only correlated with OS in patients who did not accept chemotherapy. Table 6 shows that high SPHK1 mRNA expression was associated with OS in all patients with or without radiotherapy, while high SPHK2 mRNA expression was only correlated with OS in 

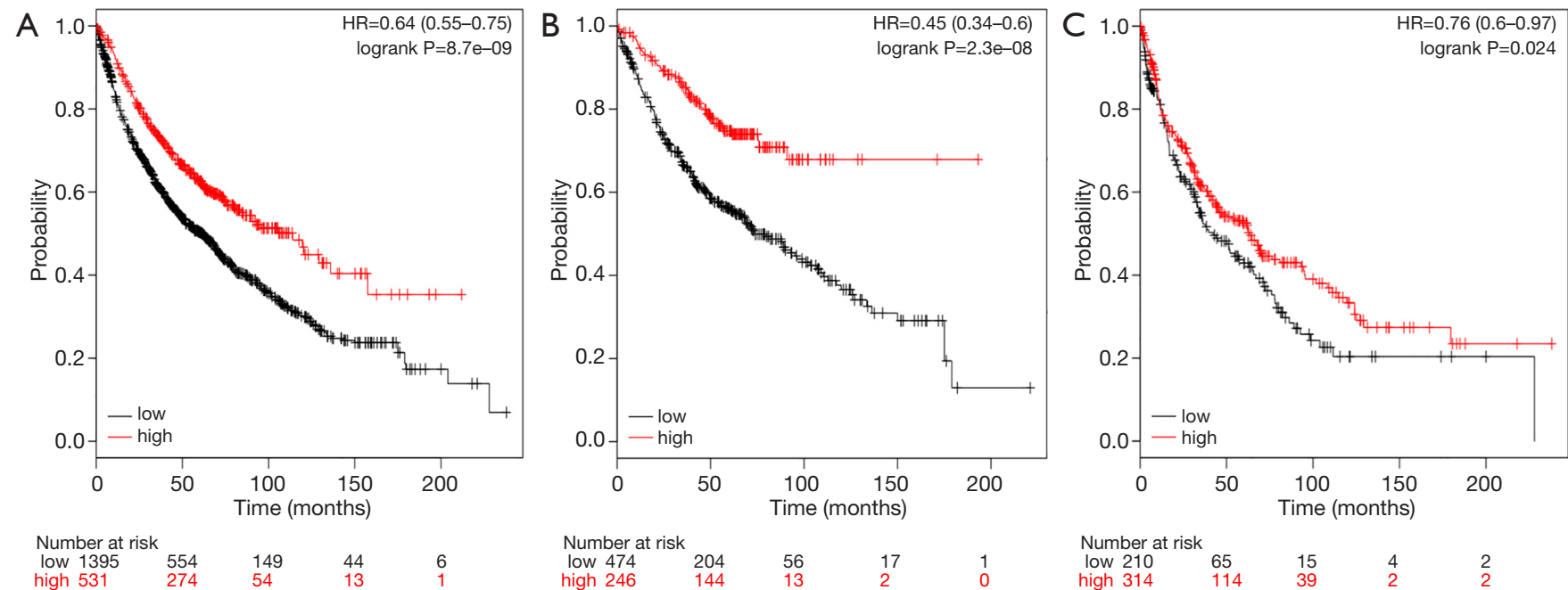

Figure 3 The prognostic role of SPGL1 mRNA level in NSCLC patients. The Affymetrix ID is 212322_at. The Kaplan-Meier survival curves are drafted for (A) all NSCLC patients ( $\mathrm{n}=1,926)$, (B) Ade patients ( $\mathrm{n}=720)$, and (C) SCC patients ( $\mathrm{n}=524)$. NSCLC, non-small cell lung cancer; Ade, adenocarcinoma; SCC, squamous cell carcinoma.

Table 1 Correlation between S1P metabolism enzyme mRNA high expression and different smoking history in NSCLC patients

\begin{tabular}{lccccc}
\hline Gene & $\begin{array}{c}\text { Smoking } \\
\text { history }\end{array}$ & Patients & $\begin{array}{c}\text { Hazard } \\
\text { ratio }\end{array}$ & $95 \% \mathrm{Cl}$ & P value \\
\hline SPHK1 & Yes & 820 & 1.74 & $(1.33-2.28)$ & $4.5 \mathrm{e}-05$ \\
& No & 205 & 2.41 & $(1.36-4.24)$ & 0.0018 \\
SPHK2 & Yes & 820 & 0.67 & $(0.54-0.83)$ & 0.00015 \\
& No & 205 & 0.62 & $(0.35-1.08)$ & 0.0872 \\
SGPL1 & Yes & 820 & 0.74 & $(0.58-0.94)$ & 0.0155 \\
& No & 205 & 0.56 & $(0.32-0.98)$ & 0.0408 \\
\hline
\end{tabular}

NSCLC, non-small cell lung cancer; S1P, sphingosine-1-phosphate.

Table 2 Correlation between S1P metabolism enzyme mRNA high expression and non-metastasis NSCLC patients

\begin{tabular}{lccccc}
\hline Gene & $\begin{array}{c}\text { AJCC } \\
\text { stage M }\end{array}$ & Patients & $\begin{array}{c}\text { Hazard } \\
\text { ratio }\end{array}$ & $95 \% \mathrm{Cl}$ & P value \\
\hline SPHK1 & 0 & 681 & 1.43 & $(1.11-1.84)$ & 0.0047 \\
SPHK2 & 0 & 681 & 0.77 & $(0.62-0.95)$ & 0.0162 \\
SGPL1 & 0 & 681 & 0.76 & $(0.59-0.97)$ & 0.0298 \\
\hline
\end{tabular}

NSCLC, non-small cell lung cancer; S1P, sphingosine-1-phosphate; AJCC, American Joint Committee on Cancer.
Table 3 Correlation between S1P metabolism enzyme mRNA high expression and the surgery success of NSCLC patients

\begin{tabular}{lccccc}
\hline Gene & $\begin{array}{c}\text { Surgery } \\
\text { success }\end{array}$ & Patients & $\begin{array}{c}\text { Hazard } \\
\text { ratio }\end{array}$ & $95 \% \mathrm{Cl}$ & P value \\
\hline SPHK1 & $\begin{array}{c}\text { Negative } \\
\text { margins }\end{array}$ & 726 & 2.03 & $(1.62-2.55)$ & $5.8 \mathrm{e}-10$ \\
SPHK2 & $\begin{array}{c}\text { Negative } \\
\text { margins }\end{array}$ & 726 & 0.57 & $(0.44-0.72)$ & $3.1 \mathrm{e}-06$ \\
SGPL1 & $\begin{array}{c}\text { Negative } \\
\text { margins }\end{array}$ & 726 & 0.7 & $(0.54-0.91)$ & 0.0071 \\
\hline
\end{tabular}

NSCLC, non-small cell lung cancer; S1P, sphingosine-1-phosphate.

Table 4 Correlation between S1P metabolism enzyme mRNA high expression and the clinical stage of NSCLC patients

\begin{tabular}{lccccc}
\hline Gene & Stage & Patients & Hazard ratio & $95 \% \mathrm{Cl}$ & P value \\
\hline SPHK1 & I & 577 & 2.14 & $(1.62-2.82)$ & $3.4 \mathrm{e}-08$ \\
& II & 244 & 0.78 & $(0.52-1.19)$ & 0.2472 \\
& III & 70 & 1.33 & $(0.71-2.5)$ & 0.3657 \\
SPHK2 & I & 577 & 0.51 & $(0.39-0.67)$ & $7.3 \mathrm{e}-07$ \\
& II & 244 & 0.56 & $(0.38-0.82)$ & 0.0023 \\
& III & 70 & 0.64 & $(0.37-1.1)$ & 0.1048 \\
SGPL1 & I & 577 & 0.52 & $(0.4-0.69)$ & $2.4 \mathrm{e}-06$ \\
& II & 244 & 0.51 & $(0.34-0.77)$ & 0.00091 \\
& III & 70 & 1.37 & $(0.8-2.36)$ & 0.2483 \\
\hline
\end{tabular}

NSCLC, non-small cell lung cancer; S1P, sphingosine-1-phosphate. 
Table 5 Correlation between S1P metabolism enzyme mRNA high expression and the chemotherapy of NSCLC patients

\begin{tabular}{llllll}
\hline \multirow{2}{*}{ Gene } & Chemotherapy & Patients & $\begin{array}{c}\text { Hazard } \\
\text { ratio }\end{array}$ & $95 \% \mathrm{Cl}$ & P value \\
\hline SPHK1 & Yes & 176 & 1.47 & $(0.96-2.24)$ & 0.0758 \\
& No & 310 & 2.06 & $(1.46-2.89)$ & $2.2 \mathrm{e}-05$ \\
\multirow{2}{*}{ SPHK2 } & Yes & 176 & 0.73 & $(0.45-1.16)$ & 0.1814 \\
& No & 310 & 0.63 & $(0.45-0.88)$ & 0.0065 \\
\multirow{2}{*}{ SGPL1 } & Yes & 176 & 0.77 & $(0.5-1.18)$ & 0.2293 \\
& No & 310 & 0.74 & $(0.5-1.09)$ & 0.1277 \\
\hline
\end{tabular}

NSCLC, non-small cell lung cancer; S1P, sphingosine-1-phosphate.

Table 6 Correlation between S1P metabolism enzyme mRNA high expression and the radiotherapy of NSCLC patients

\begin{tabular}{lcrrrl}
\hline \multirow{2}{*}{ Gene } & Radiotherapy & Patients & $\begin{array}{c}\text { Hazard } \\
\text { ratio }\end{array}$ & $95 \% \mathrm{Cl}$ & P value \\
\hline SPHK1 & Yes & 70 & 1.86 & $(1.03-3.37)$ & 0.0364 \\
& No & 271 & 1.55 & $(1.09-2.21)$ & 0.015 \\
\multirow{2}{*}{ SPHK2 } & Yes & 70 & 0.6 & $(0.33-1.1)$ & 0.0971 \\
& No & 271 & 0.67 & $(0.47-0.95)$ & 0.0251 \\
\multirow{2}{*}{ SGPL1 } & Yes & 70 & 1.46 & $(0.8-2.65)$ & 0.2104 \\
& No & 271 & 0.69 & $(0.44-1.06)$ & 0.0865 \\
\hline
\end{tabular}

NSCLC, non-small cell lung cancer; S1P, sphingosine-1-phosphate.

patients who accepted radiotherapy.

Cox multivariate regression analysis was performed with selected clinical parameters, including cancer grade, stage, gender, and smoking history. However, the full clinical data were only available for 130 SCC patients in the multivariate analysis. In accordance with univariate analysis, neither high SPHK1 expression (HR 0.83, 95\% CI: 0.51-1.36, $\mathrm{P}=0.4636$ ) nor high SPHK2 expression (HR 0.69, 95\% CI: $0.41-1.18, \mathrm{P}=0.1732$ ) was significantly associated with worse OS in SCC patients. Unexpectedly, high SPGL1 gene expression was associated with worse OS in SCC patients (HR 1.77, 95\% CI: 1.03-3.04, $\mathrm{P}=0.0388$ ).

\section{Discussion}

The KM plotter is a widely-used online tool for assessing associations between gene expression and outcomes in patients with different cancers. Numerous genes were identified as prognostic biomarkers and therapeutic targets by the KM plotter, such as $M M P 1, C B X 1$, and RPS14 in breast cancer (14-16); E2F2 and CCNE2 in ovarian cancer (19); TREM2 in gastric cancer (12); and Notch1-3 and HSPB1 in NSCLC $(23,24)$. With this online tool, we propose SPHK1, $S P H K 2$, and SGPL1 mRNA levels as potential prognostic predictors for NSCLC patients. For lack of clinical data, whether these mRNA levels are independent prognostic factors in NSCLC is still not determined.

$\mathrm{S} 1 \mathrm{P}$ has a pivotal role in cell proliferation, migration, and inflammation. The accumulation of S1P is a balance between the synthesis catalyzed by SPHKs and the catabolism catalyzed by SGPL1 $(4,8)$. Aberrant S1P metabolism and dysregulation of its downstream signals were observed in several tumors including breast, esophageal, prostate, colon, oral cancer, and NSCLC (4,26-30).

SPHK1 was elevated in various types of cancers, contributing to tumor proliferation, invasion and metastasis $(31,32)$. In the human A549 NSCLC cell line, SPHK1 induced cell proliferation and suppressed cell apoptosis through nuclear factor- $\kappa \mathrm{B}(\mathrm{NF}-\kappa \mathrm{B})$ and intracellular calcium signaling (33). The invasion and migration capacities of NSCLC cell were also enhanced by SPHK1, with the promoted epithelial mesenchymal transition (EMT), activation of the PI3K-Akt pathway, and E-cadherin expression $(34,35)$. In accordance with the experimental results, clinical evidence revealed an approximate 2 -fold elevation of SPHK1 in NSCLC tissue versus normal tissue $(10,36)$. In addition, high SPHK1 expression was markedly associated with advanced clinical staging, tumor-nodemetastasis (TNM) classification, and worse OS; and SPHK1 level was identified as an independent prognostic factor for NSCLC patients (10). Consistent with previous studies, our results indicate that high SPHK1 mRNA expression was significantly associated with worse OS in all NSCLC patients. Moreover, our stratified analysis showed that SPHK1 mRNA might be more efficient in predicting outcomes in Ade patients than SCC patients.

SPHK2 is also widely believed to be an oncogene in lung cancer. Inhibition of SPHK2 in A549 human NSCLC cells led to cell apoptosis in previous research (37). Another recent study suggests that SPHK2-generating S1P binds to human telomerase reverse transcriptase in NSCLC cells, promoting telomerase stability, cell proliferation, and tumor growth. The level of SPHK2 was gradually upregulated from normal, metaplasia/dysplasia tissues to NSCLC tissues (9). Additionally, the level of SPHK2 in NSCLC tissues was significantly associated with the proliferative index, lymph node status, histology grade, and 
clinical stage (9). High SPHK2 expression was regarded as an independent prognostic factor for worse OS in NSCLC patients (9). Contrary to previous findings, our results show that high SPHK2 mRNA expression was strongly linked to better OS in NSCLC patients. This diversity can probably be explained by some regulators between $S P H K 2$ mRNA expression and SPHK2. For example, microRNA promotes target mRNA degradation or silencing by binding to 3'-untranslated regions (38). It was reported that miR-338-3p suppressed NSCLC progression in nude mouse xenograft models by downregulating SPHK2 (39). However, it was observed that miR-338-3p expression was also downregulated in NCCLC. Thus, the regulatory mechanism between SPHK2 mRNA expression and SPHK2 protein level in NSCLC needs further investigation.

SGPL1, the only enzyme that irreversibly degrades S1P in cells, was suggested as a potential tumor suppressor. SPGL1 deficiency has been shown to lead to increased cell growth, cell proliferation and transformation. Moreover, loss of SGPL1 causes cell resistance to apoptosis induced by chemotherapy, accompanied by a strong increase in Bcl-2 and Bcl-xL protein levels (40). Compared with control animals, mice with intestinal epithelium-specific Sgpl1 deletion exhibited greater disease activity, cytokine levels, S1P accumulation, tumors, STAT3 activation in a chemically induced tumor model. Mechanically, silencing SPGL1 promoted tumorigenic transformation through a pathway involving extracellular transport of S1P through S1P transporter Spns2, S1P receptor activation, and JAK2/STAT3-dependent pathway (41). Compared with normal tissues, the levels of SGPL1 were lower in human melanoma, colon carcinoma, oral cancer tissues and prostate cancer (27-29). In human prostatectomy specimens, a marked decrease in SPL enzymatic activity was observed in tumor samples, compared with normal tissue. Tissue microarray analysis confirmed that the loss of SPGL1 in human prostate cancer was associated with a higher Gleason score (27). However, the role of SGPL1 in NSCLC remains unclear. Our univariate analysis shows that high SGPL1 mRNA expression was significantly associated with better survival in all NSCLC patients. However, in our multivariate analysis, SCC patients with high SGPL1 mRNA expression were associated with worse outcomes, suggesting SGPL1 is not an independent prognostic factor in SCC. Further clinical research is advocated in this area.

Evidence suggests that targeting enzymes in S1P metabolism makes NSCLC cells sensitized to radiation or chemotherapy (6,27). SPHK1-overexpressed A549 NSCLC cells had a higher survival rate compared with control cells after treatment of doxorubicin or docetaxel (10). The knocking down of SPHK1 with small interfering RNA (siRNA) not only inhibited cancer cell proliferation but also sensitized cancer cells to apoptosis induced by doxorubicin or docetaxel (10). SPHK1 inhibitor, in combination with chemotherapy, significantly reduced the tumor size and weight of xenografted NSCLC tumors in mice (10). Notably, SPHK2 was implicated in promoting cell apoptosis and sensitivity to treatment of gefitinib in NSCLC (42). Proposed as a radio-responsive protein, SGPL1 potentiated cell death after ironizing radiation (43). It was also reported that SGPL1 sensitized lung cancer cells to cisplatin, mainly mediated by upregulation of p38 (44). Collectively, these findings indicate that targeting different S1P metabolism enzymes might improve the efficacy of different chemotherapeutic drugs or radiation. The mRNA levels of S1P metabolism enzymes in NSCLC might provide an indication for which chemotherapeutic drugs to choose in clinical practice. The combination of SPHK1 inhibitor and SGPL1 activator would be a new therapeutic strategy for chemo- or chemo-resistant NSCLC.

\section{Conclusions}

In NSCLC patients, high SPHK1 mRNA expression was strongly associated with worse survival. In contrast, high SPHK2 and SGPL1 mRNA expression was an indicator of better survival. The combination of SPHK1 inhibitor and SGPL1 activator might be a new therapeutic strategy for patients with NSCLC resistant to chemo- or radio-therapy.

\section{Acknowledgments}

Funding: This work was supported by the China National Natural Science Foundation (NSFC-81400047), the Natural Science Foundation of Jiangsu Province (grant nos. BK20150213 and BK20161155), and Jiangsu Provincial Key Medical Discipline, The project of Invigorating Health Care through Science, Technology, and Education (no. ZDXKA2016014). The funders had no role in the study design, data collection, and analysis, decision to publish, or preparation of the manuscript.

\section{Footnote}

Conflicts of Interest: The authors have no conflicts of interest 
to declare.

Ethical Statement: The authors are accountable for all aspects of the work in ensuring that questions related to the accuracy or integrity of any part of the work are appropriately investigated and resolved.

\section{References}

1. Ferlay J, Soerjomataram I, Dikshit R, et al. Cancer incidence and mortality worldwide: sources, methods and major patterns in GLOBOCAN 2012. Int J Cancer 2015;136:E359-86.

2. Schiller JH, Harrington D, Belani CP, et al. Comparison of four chemotherapy regimens for advanced non-smallcell lung cancer. N Engl J Med 2002;346:92-8.

3. Wu X, Ruan L, Yang Y, et al. Analysis of gene expression changes associated with human carcinoma-associated fibroblasts in non-small cell lung carcinoma. Biol Res 2017;50:6.

4. Ebenezer DL, Fu P, Natarajan V. Targeting sphingosine1-phosphate signaling in lung diseases. Pharmacol Ther 2016;168:143-57.

5. Vishwakarma S, Agarwal R, Goel SK, et al. Altered Expression of Sphingosine-1-Phosphate Metabolizing Enzymes in Oral Cancer Correlate With Clinicopathological Attributes. Cancer Invest 2017;35:139-41.

6. Yester JW, Tizazu E, Harikumar KB, et al. Extracellular and intracellular sphingosine-1-phosphate in cancer. Cancer Metastasis Rev 2011;30:577-97.

7. Bradley E, Dasgupta S, Jiang X, et al. Critical role of Spns2, a sphingosine-1-phosphate transporter, in lung cancer cell survival and migration. PLoS One 2014;9:e110119.

8. Furuya H, Shimizu Y, Kawamori T. Sphingolipids in cancer. Cancer Metastasis Rev 2011;30:567-76.

9. Wang Q, Li J, Li G, et al. Prognostic significance of sphingosine kinase 2 expression in non-small cell lung cancer. Tumour Biol 2014;35:363-8.

10. Song L, Xiong H, Li J, et al. Sphingosine kinase-1 enhances resistance to apoptosis through activation of $\mathrm{PI} 3 \mathrm{~K} / \mathrm{Akt} / \mathrm{NF}-\mathrm{kappaB}$ pathway in human non-small cell lung cancer. Clin Cancer Res 2011;17:1839-49.

11. Lánczky A, Nagy Á, Bottai G, et al. miRpower: a web-tool to validate survival-associated miRNAs utilizing expression data from 2178 breast cancer patients. Breast Cancer Res Treat 2016;160:439-46.
12. Zhang $\mathrm{X}$, Wang $\mathrm{W}$, Li $\mathrm{P}$, et al. High TREM2 expression correlates with poor prognosis in gastric cancer. Hum Pathol 2018;72:91-9.

13. Kumar D, Hassan MK, Pattnaik N, et al. Reduced expression of IQGAP2 and higher expression of IQGAP3 correlates with poor prognosis in cancers. PLoS One 2017;12:e0186977.

14. Wang J, Ye C, Lu D, et al. Matrix metalloproteinase-1 expression in breast carcinoma: a marker for unfavorable prognosis. Oncotarget 2017;8:91379-90.

15. Liang YK, Lin HY, Chen CF, et al. Prognostic values of distinct CBX family members in breast cancer. Oncotarget 2017;8:92375-87.

16. Fang E, Zhang X. Identification of breast cancer hub genes and analysis of prognostic values using integrated bioinformatics analysis. Cancer Biomark 2017;21:373-81.

17. Tuo YL, Ye YF. MGP is downregulated due to promoter methylation in chemoresistant ER+ breast cancer and high MGP expression predicts better survival outcomes. Eur Rev Med Pharmacol Sci 2017;21:3871-8.

18. Bai $\mathrm{Y}$, Qiao L, Xie N, et al. Expression and prognosis analyses of the Tob/BTG antiproliferative (APRO) protein family in human cancers. PLoS One 2017;12:e0184902.

19. Xie L, Li T, Yang LH. E2F2 induces MCM4, CCNE2 and WHSC1 upregulation in ovarian cancer and predicts poor overall survival. Eur Rev Med Pharmacol Sci 2017;21:2150-6.

20. Tian X, Han Y, Yu L, et al. Decreased expression of ALDH5A1 predicts prognosis in patients with ovarian cancer. Cancer Biol Ther 2017;18:245-51.

21. Chen C, Wang X, Huang S, et al. Prognostic roles of Notch receptor mRNA expression in human ovarian cancer. Oncotarget 2017;8:32731-40.

22. Ortega CE, Seidner Y, Dominguez I. Mining CK2 in cancer. PLoS One 2014;9:e115609.

23. Xiong J, Zhang X, Chen X, et al. Prognostic roles of mRNA expression of notch receptors in non-small cell lung cancer. Oncotarget 2017;8:13157-65.

24. Huang ZC, Li H, Sun ZQ, et al. Distinct prognostic roles of HSPB1 expression in non-small cell lung cancer. Neoplasma 2018;65:161-6.

25. Györffy B, Surowiak P, Budczies J, et al. Online survival analysis software to assess the prognostic value of biomarkers using transcriptomic data in non-small-cell lung cancer. PLoS One 2013;8:e82241.

26. Wang YC, Tsai CF, Chuang HL, et al. Benzyl butyl phthalate promotes breast cancer stem cell expansion via SPHK1/S1P/S1PR3 signaling. Oncotarget 
2016;7:29563-76.

27. Brizuela L, Ader I, Mazerolles C, et al. First evidence of sphingosine 1-phosphate lyase protein expression and activity downregulation in human neoplasm: implication for resistance to therapeutics in prostate cancer. Mol Cancer Ther 2012;11:1841-51.

28. Oskouian B, Sooriyakumaran P, Borowsky AD, et al. Sphingosine-1-phosphate lyase potentiates apoptosis via $\mathrm{p} 53$ - and $\mathrm{p} 38$-dependent pathways and is downregulated in colon cancer. Proc Natl Acad Sci U S A 2006;103:17384-9.

29. Patmanathan SN, Johnson SP, Lai SL, et al. Aberrant expression of the S1P regulating enzymes, SPHK1 and SGPL1, contributes to a migratory phenotype in OSCC mediated through S1PR2. Sci Rep 2016;6:25650.

30. Pan J, Tao YF, Zhou Z, et al. An novel role of sphingosine kinase-1 (SPHK1) in the invasion and metastasis of esophageal carcinoma. J Transl Med 2011;9:157.

31. Zhang Y, Wang Y, Wan Z, et al. Sphingosine kinase 1 and cancer: a systematic review and meta-analysis. PLoS One 2014;9:e90362.

32. Marfe G, Mirone G, Shukla A, et al. Sphingosine kinases signalling in carcinogenesis. Mini Rev Med Chem 2015;15:300-14.

33. Chen K, Pan Q, Gao Y, et al. DMS triggers apoptosis associated with the inhibition of SPHK1/NF-kappaB activation and increase in intracellular $\mathrm{Ca} 2+$ concentration in human cancer cells. Int J Mol Med 2014;33:17-24.

34. Zhu L, Wang Z, Lin Y, et al. Sphingosine kinase 1 enhances the invasion and migration of non-small cell lung cancer cells via the AKT pathway. Oncol Rep 2015;33:1257-63.

35. Ni M, Shi XL, Qu ZG, et al. Epithelial mesenchymal transition of non-small-cell lung cancer cells A549 induced by SPHK1. Asian Pac J Trop Med 2015;8:142-6.

36. Johnson KR, Johnson KY, Crellin HG, et al. Immunohistochemical distribution of sphingosine kinase 1 in normal and tumor lung tissue. J Histochem Cytochem 2005;53:1159-66.

37. Guan S, Liu YY, Yan T, et al. Inhibition of ceramide glucosylation sensitizes lung cancer cells to ABC294640, a first-in-class small molecule SphK2 inhibitor. Biochem Biophys Res Commun 2016;476:230-6.

38. Hale BJ, Yang CX, Ross JW. Small RNA regulation of reproductive function. Mol Reprod Dev 2014;81:148-59.

39. Zhang G, Zheng H, Zhang G, et al. MicroRNA-338-3p suppresses cell proliferation and induces apoptosis of nonsmall-cell lung cancer by targeting sphingosine kinase 2 . Cancer Cell Int 2017;17:46.

40. Colié S, Van Veldhoven PP, Kedjouar B, et al. Disruption of sphingosine 1-phosphate lyase confers resistance to chemotherapy and promotes oncogenesis through Bcl-2/ Bcl-xL upregulation. Cancer Res 2009;69:9346-53.

41. Degagné E, Pandurangan A, Bandhuvula $\mathrm{P}$, et al. Sphingosine-1-phosphate lyase downregulation promotes colon carcinogenesis through STAT3-activated microRNAs. J Clin Invest 2014;124:5368-84.

42. Liu W, Ning J, Li C, et al. Overexpression of Sphk2 is associated with gefitinib resistance in non-small cell lung cancer. Tumour Biol 2016;37:6331-6.

43. Kumar A, Oskouian B, Fyrst H, et al. S1P lyase regulates DNA damage responses through a novel sphingolipid feedback mechanism. Cell Death Dis 2011;2:e119.

44. Min J, Van Veldhoven PP, Zhang L, et al. Sphingosine1-phosphate lyase regulates sensitivity of human cells to select chemotherapy drugs in a p38-dependent manner. Mol Cancer Res 2005;3:287-96.

Cite this article as: Wang Y, Shen Y, Sun X, Hong TL, Huang LS, Zhong M. Prognostic roles of the expression of sphingosine-1-phosphate metabolism enzymes in non-small cell lung cancer. Transl Lung Cancer Res 2019;8(5):674-681. doi: 10.21037/tlcr.2019.10.04 